\title{
Änderung TARMED-Browser Version 1.05/1.05.03 - ein Ärgernis!
}

Dr. med. Ernst Gähler, Mitglied des Zentralvorstandes der FMH, Leiter Ressort Tarife und Verträge
Die Einführung des TARMED-Browsers Version 1.05/1.05.03 hat bei Ihnen verständlicherweise viel Ärger ausgelöst. Die Kurzfristigkeit der Änderungen ist sowohl für alle Anwenderinnen und Anwender als auch für die FMH eine Zumutung. Wie kam es dazu, und wo liegen die Probleme?

Der Bundesrat hat die Version 1.05/1.05.03 erst am 21. November 2007 genehmigt, wodurch eine verspätete Umsetzung unausweichlich war. Aufgrund der Übergangslösung für die Notmassnahmen im Bereich Radiologie, die bis zum 31. Dezember 2007 terminiert waren, musste eine Einführung per 1. Januar 2008 erfolgen.

Dabei hatte alles termingerecht begonnen: Wie Sie wissen, waren wir von der Arbeitsgruppe RE III Phase 2 gezwungen, die umfangreichen Arbeiten bis Ende Mai 2007 abzuschliessen. Dies gelang auch dank eines enormen Efforts der Arbeitsgruppe der FMH sowie der übrigen Partner santésuisse, MTK und H+. So konnte unsere Arbeitsgruppe den Schlussbericht mit allen Anträgen fristgerecht beim BAG einreichen.

Ab 1. Juni 2007 lag der Ball somit beim BAG bzw. beim Bundesrat. Während insgesamt mehr als fünf Monaten lag der Antrag bei den Behörden, ohne dass diese etwas unternommen haben, bis sie unseren Schlussbericht schliesslich am 21. November 2007 genehmigten. Eine solche Verzögerung ist für uns nicht akzeptabel. Die FMH hat deshalb im Leitungsgremium TARMED
Suisse diesen Herbst den Antrag gestellt, die TARMED-Version 1.05 auf jeden Fall ab 1. Januar 2008 einzuführen. Diesem Antrag wurde stattgegeben. Die definitive Qualitätskontrolle (Sumex) der Version 1.05 konnte somit erst nach der Genehmigung durch den Bundesrat stattfinden mit Folgen: Sowohl die Kostenträger als auch die Leistungserbringer haben nach der Publikation der Version 1.05 verschiedene Fehler und Unklarheiten festgestellt. Diese betrafen vor allem das Kapitel 39 «Bildgebende Verfahren». Diese und weitere Fehler wurden umgehend und mit ausserordentlichem Einsatz der FMH und der Partner im Rahmen einer Fehlerkonferenz behoben, um so die Funktionalität der TARMED-Tarifdatenbank zu gewährleisten. Damit war die Version 1.05/1.05.03 geschaffen, die seit dem 4. Januar 2008 Gültigkeit hat; weitere Fehler wurden in der Fehlerkonferenz am 10. Januar 2008 korrigiert.

Wegen der verspäteten Genehmigung durch den Bundesrat erfolgte die Auslieferung des TARMED-Browsers Version 1.05/1.05.03 erst kürzlich. Dafür entschuldigt sich die FMH in aller Form und protestiert mit Vehemenz gegen die Verzögerungen der Genehmigung durch die Behörden, zumal alle Partner - die FMH, santésuisse, H+ und MTK - ihre Arbeiten termingerecht eingereicht hatten. 